\title{
¿Qué es la Cultura? Sistemas de Gente, Lugares, y Prácticas
}

\author{
José M. Causadias, $\mathrm{PhD}$ \\ School of Social and Family Dynamics \\ Arizona State University
}

\begin{abstract}
Nota del Autor:
Cualquier comentario en relación a este articulo debe ser dirigida a José M. Causadias, School of Social and Family Dynamics, Arizona State University, Cowden Family Resources Building, 850 South Cady Mall, Tempe, AZ 85281. Teléfono: 480-727-4358; Fax: 480-965-6779; E-mail: jose.causadias@asu.edu ORCID: https://orcid.org/0000-0001-8249-5497
\end{abstract}

Este documento es una traducción al castellano realizada por el autor de la publicación original en inglés: Causadias, J. M. (2020). What is culture? Systems of people, places, and practices. Applied Developmental Science, 24(4), 310-322. Agradezco a Rodrigo Cárcamo y Juan Causadias por sus sugerencias y comentarios sobre esta traducción.

Este trabajo fue apoyado mediante financiamiento durante el verano de 2019 por el Latino Resilience Enterprise y la School of Social and Family Dynamics, Arizona State University. No recibí ningún otro apoyo financiero de agencias públicas, comerciales, o no gubernamentales en la realización de esta publicación. Declaro que no tengo conflictos de interés. 


\section{Resumen}

La cultura es un concepto difuso sin límites fijos, teniendo un significado diferente de acuerdo a cada situación. Para enfrentar este problema, propongo un modelo- $p$ para entender la cultura como un sistema de gente, lugares, y prácticas, con un propósito como afianzar, justificar, o resistir el poder. La gente hace referencia a dinámicas poblacionales, relaciones sociales, y cultura en grupos. Los lugares hacen referencia a dinámicas ecológicas, influencias institucionales, y cultura en contextos. Las prácticas hacen referencia a las dinámicas participativas, implicación comunitaria, y cultura en acción. El poder hace referencia a forzar a otros a obedecer (poder-sobre la gente), controlar el acceso a espacios (poder-en lugares), y actuar como es deseado (poder-para practicar). Uso el ejemplo del racismo para ilustrar el modelo- $p$ y proponer aplicaciones en teoría, investigación, e intervención en ciencias aplicadas del desarrollo humano.

Palabras clave: cultura; sistema; gente; lugares; prácticas; poder. 


\section{¿Qué es la Cultura? Sistemas de Gente, Lugares, y Prácticas}

¿Es hora de abandonar el concepto de la cultura? La respuesta es afirmativa, de acuerdo con varios académicos que ven este concepto como un obstáculo para el progreso científico. El término cultura se ha comparado al protoplasma, una caja negra con cualidades imprecisas pretendiendo ofrecer explicaciones (Tooby, 2015), una palabra de siete letras equivalente a dios (Betzig, 2015), denunciado como un concepto conveniente para designar todo tipo de cosas que sentimos deben ser agrupadas juntas (Boyer, 2015). En realidad, la cultura es un concepto difuso, un término sin límites fijos, teniendo un significado diferente de acuerdo a cada situación (Gjerde, 2004; Spencer-Oatey \& Franklin, 2012). A diferencia de los conceptos crujientes que tienen un significado relativamente fijo, propiedades establecidas, y límites estables; los conceptos difusos tienen varias capas de significado, cambiando su sentido de acuerdo al contexto (Haack, 1996).

Esta característica es la principal razón por la que es tan difícil definir la cultura. De hecho, hay una larga tradición de revisiones de las definiciones de la cultura (Kroeber \& Kluckhohn, 1952; Lonner \& Malpass, 1994), y de denunciar la confusión en la conceptualización de la cultura como un problema central para la psicología (Cooper \& Denner, 1998), y otras ciencias sociales (Durham, 1991). Es posible el progreso de la ciencia sin un consenso sobre la definición de la cultura, pero especificar sus características principales puede ser útil en acelerar estos avances (Betancourt \& Lopez, 1993).

Mejores definiciones de la cultura pueden ser útiles para evitar prácticas de investigación cuestionables, como tratar la cultura como un factor estático resistente al cambio o simplemente como variable de control en análisis estadísticos (Quintana et al., 2006), usar la cultura para promover modelos deficitarios que presentan grupos humanos como esencialmente inferiores y 
en riesgo (García Coll et al., 1996), asumir que hay grandes diferencias culturales entre grupos sin ninguna justificación teórica (Gjerde, 2004), y menospreciar el rol normativo y multifacético de los procesos culturales en el desarrollo humano (Rogoff, 2003). Por estos motivos, mejorar las definiciones de la cultura es fundamental para evitar los sesgos y malentendidos que influyen la teoría, investigación, e intervención en ciencias aplicadas del desarrollo humano.

Para promover una mejor ciencia, propongo un modelo- $p$ en el cual defino la cultura como un sistema de gente, lugares, y prácticas, con un propósito como afianzar, justificar, o resistir el poder. La gente hace referencia a dinámicas poblacionales, relaciones sociales, y cultura en grupos. Los lugares hacen referencia a dinámicas ecológicas, influencias institucionales, y cultura en contextos. Las prácticas hacen referencia a las dinámicas participativas, implicación comunitaria, y cultura en acción. El poder hace referencia a forzar a otros a obedecer (podersobre la gente), controlar el acceso a espacios (poder-en lugares), y actuar como es deseado (poder-para practicar).

Seguidamente, uso el ejemplo del racismo para ilustrar el modelo- $p$ y proponer aplicaciones en teoría, investigación, e intervención en ciencias aplicadas del desarrollo humano. En este artículo, incorporo elementos de la epistemología relacional (Overton, 2010, 2015), la psicopatología del desarrollo (Cicchetti, 1984), el modelo integrativo para el estudio de las competencias del desarrollo en niños de grupos minoritarios (García Coll et al., 1996), distintas tradiciones de investigación cultural en psicología (Shweder, 2000), la teoría racial crítica (Bonilla-Silva, 2010), y la teoría relacional dinámica del poder (Roscigno, 2011). Este artículo está enmarcado en la teoría y la investigación en los Estados Unidos de América. Uso el concepto de modelo porque lo que presento aquí es el inicio de una conceptualización más comprensiva sobre la cultura. 


\section{¿Qué es la Cultura? Introduciendo el modelo-p}

La cultura es un sistema, un todo dinámico que crea y es creado por gente, lugares, y prácticas (Figura 1). El sistema y sus componentes son inseparables y están dedicados a la determinación mutua: el todo organiza las partes y las partes organizan el todo (Overton, 2010). La gente crea la cultura mediante prácticas compartidas en lugares, y la cultura moldea la manera en que la gente se involucran en prácticas y construyen lugares. El modelo- $p$ es consistente con una rica tradición que define la cultura como un sistema (Triandis, 2007). Por ejemplo, Tylor (1871) definió la cultura como "ese todo complejo que incluye conocimientos, creencias, arte, morales, costumbres y cualquier otras capacidades y hábitos adquiridos por el hombre como miembro de la sociedad" (p. 1). Geertz (1973) definió la religión como un sistema cultural de símbolos que influye las acciones, el orden social, y la visión del mundo. Pero para comprender como la cultura funciona como un sistema hace falta discutir sus componentes.

La gente es el primer componente esencial de la cultura como un sistema: no hay cultura sin gente y no hay gente sin cultura. La gente hace referencia a dinámicas poblacionales, relaciones sociales, y cultura en grupos, incluyendo familias, comunidades, y naciones. Muchas definiciones de la cultura enfatizan este componente del sistema (Cooper \& Denner, 1998). Por ejemplo, Snowdon (2018) definió la cultura como patrones de conducta que tiene cierta continuidad a través de las generaciones entre grupos específicos o poblaciones, variando entre gente diferente, pero manteniendo cierta continuidad (Snowdon, 2018). Los antropólogos han descrito la cultura como sistemas de creencias, ideales, comportamiento, y tradiciones relacionadas a poblaciones específicas (Rogoff, 2003; Shweder, 2000).

La investigación de la cultura con frecuencia se enfoca en temas relacionados con gente, incluyendo la variación dentro de poblaciones (diversidad cultural) y las diferencias entre grupos 
(diferencias culturales). De acuerdo con Shweder (2000), el estudio de la cultura en psicología se puede clasificar en campos especializados a partir de las estrategias que emplean para entender a la gente, ya sea a partir de enfatizar la cultura como fuente de diversidad (psicología cultural), las experiencias culturales únicas de ciertos grupos (psicología indígena), o los aspectos compartidos de las poblaciones humanas a pesar de la cultura (psicología transcultural). Un cuarto campo especializado se centra en las minorías raciales y étnicas (psicología de las minorías étnicas; Sue, 2009).

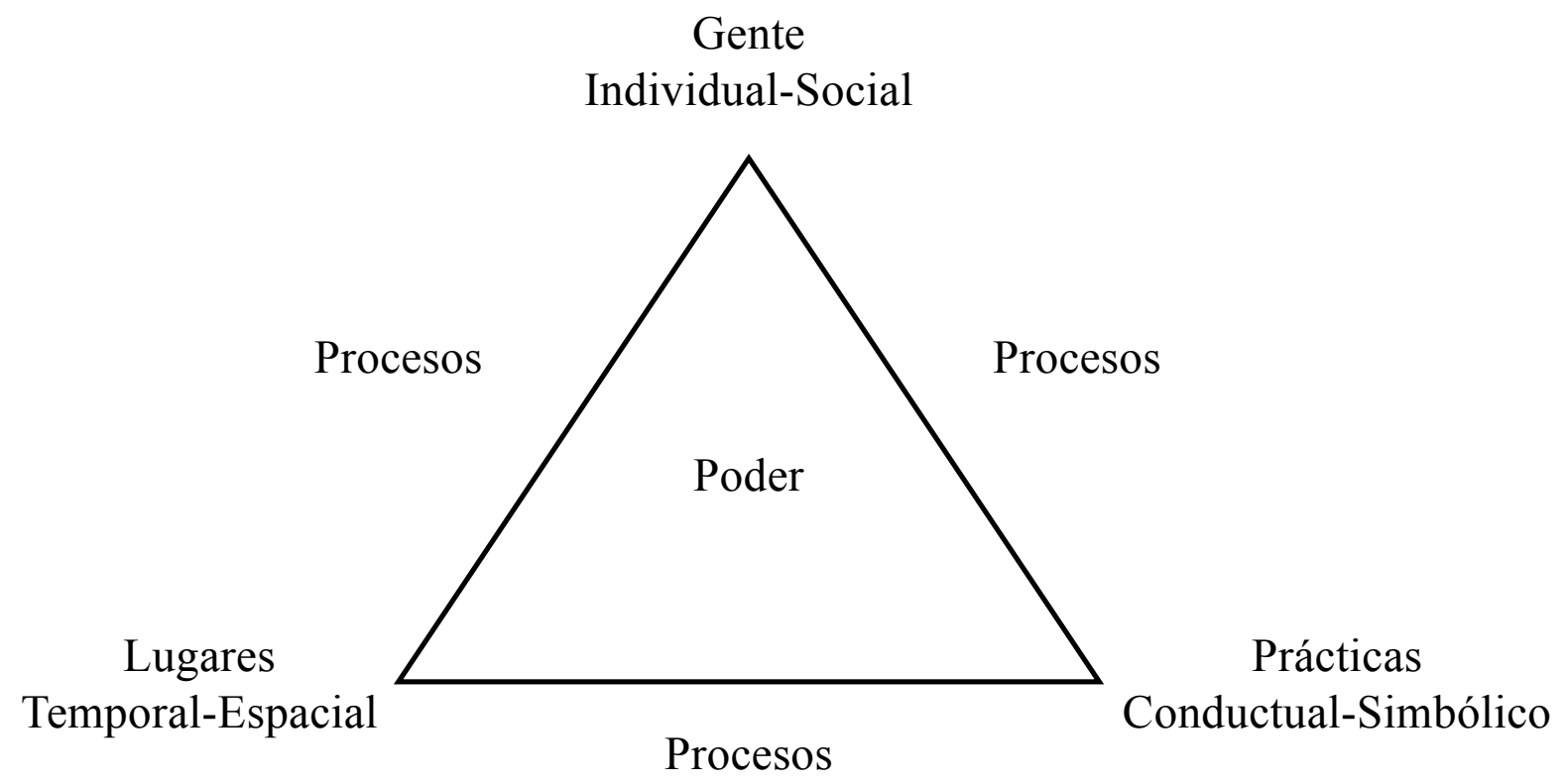

Figura 1. La cultura como sistema de gente, lugares, y prácticas conectadas por dimensiones y procesos, con un propósito como justificar o resistir el poder.

\section{(c) (i)}

Esta figura creada por José M. Causadias tiene una licencia bajo Creative Commons Attribution 4.0 International License. Los permisos que van más allá del marco de esta licencia pueden estar disponibles en https://www.josecausadias.com/contact. 
Los lugares son el segundo componente esencial de la cultura como sistema: no hay cultura sin lugares ni lugares sin cultura. Los lugares hacen referencia a dinámicas ecológicas, influencias institucionales, y cultura en contextos, incluyendo hogares, vecindarios, escuelas, y ciudades. Muchas definiciones de la cultura enfatizan este componente del sistema (Bronfenbrenner, 1979, 2005). Hay una larga tradición de enfatizar la cultura como lugares en antropología, por ejemplo, enmarcando la cultura como lugares para aprender y pensar (Cole, Gay, Glick, \& Sharp, 1971). Super and Harkness (1986) destacaron la importancia del nicho del desarrollo como un contexto único para el desarrollo individual.

La investigación de la cultura con frecuencia se enfoca en temas relacionados con lugares, incluyendo las influencias ecológicas en el desarrollo infantil, los ambientes educativos formales e informales que moldean la conducta y la cognición, y las situaciones que facilitan la adaptación o maladaptación. En psicología, el principal enfoque de cultura-como-lugar es el modelo conceptual de Bronfenbrenner, personificado en la idea que "todo depende" del contexto (Cole, 1979). La palabra “contexto" puede tomar una variedad de significados, incluyendo los entornos sociales, culturales, y ambientales. En este artículo, el contexto representa ambientes ecológicos, físicos, organizaciones, institucionales, y virtuales.

Las prácticas son el tercer componente esencial de la cultura como sistema: no hay cultura sin prácticas ni prácticas sin cultura. Las prácticas hacen referencia a las dinámicas participativas, implicación comunitaria, y cultura en acción, incluyendo enseñanza, aprendizaje, y participación en actividades cotidianas, tradiciones, y rituales. Varias conceptualizaciones de la cultura enfatizan este componente del sistema, especialmente aquellas que llevan la impronta del trabajo de Vygotsky (Rogoff, 2003). Hay un rico legado de enfoques que enfatizan cultura como prácticas en antropología, a través de amplias investigaciones etnográficas acerca de la 
participación de los niños en actividades rutinarias que influyen en su desarrollo (Alcalá, Rogoff, Mejía-Arauz, Coppens, \& Dexter, 2014).

La investigación de la cultura con frecuencia se enfoca en temas relacionados con prácticas, incluyendo la forma en que los padres enseñan a sus hijos acerca de los roles sociales y los valores (socialización cultural), como los educan acerca de su pertenencia a grupos (socialización étnica), y lo que dicen o mantienen en silencio acerca de las razas y el racismo (socialización racial). Estas prácticas pueden facilitar el riesgo, la protección, y la promoción de la salud y el bienestar. Por ejemplo, el cuidado parental positivo, la participación comunitaria, y la socialización bicultural de los Latinos en los Estados Unidos se han relacionado con el bienestar (Fuller \& García Coll, 2010).

En conclusión, la cultura es un concepto difuso, lo que hace que sea difícil definirla. El modelo-p entiende la cultura como un sistema que emerge de sus componentes, y unos componentes que crean el sistema. Creo que este enfoque puede ser útil en hacer el concepto de la cultura menos difuso y más crujiente.

\section{¿Cómo Funciona la Cultura? Conectando el modelo-p}

¿Cuál es el pegamento que une gente-lugares-prácticas en un sistema? Yo propongo que los procesos y dimensiones culturales conectan estos componentes en un todo unificado (Figura 1). Ellos juegan un papel central en la continuidad y el cambio en los sistemas culturales.

Primero, la teoría y la investigación de la cultura como gente refleja la tensión entre el individuo y el grupo (Overton \& Müller, 2012). Muchas conceptualizaciones de la cultura subrayan su naturaleza colectiva, compuesta de conductas y significados sociales (Kitayama \& Uskul, 2011). Por ende, la cultura no es simplemente un rasgo de personalidad característico de 
un individuo, sino que tiene una naturaleza supraindividual. La cultura es creada, compartida, y actualizada por grupos (Causadias, 2013). En resumen, la cultura es social.

Al mismo tiempo, la cultura es individual. La sociedad forma y es formada por individuos, reflejando un ciclo de construcción mutua (Markus \& Kitayama, 2010). Gjerde (2004) argumentó a favor de un estudio de la cultura que reconozca la agencia y la individualidad, y advirtió en contra del esencialismo: el asumir que los grupos son cohesivos, coherentes, y que tienen una naturaleza o esencia central. El esencialismo conduce al descuido de las diferencias individuales y a una visión de las personas como ‘copias de carbón’ (Gjerde, 2004). En los Estados Unidos, esto es ilustrado por la tendencia a ver a las minorías raciales y étnicas como miembros de grupos cuya conducta es moldeada principalmente por la cultura, y menos influida por características individuales como la personalidad en comparación a la gente blanca (Causadias, Vitriol, \& Atkin, 2018).

La dimensión individual-social de la cultura reconcilia esta tensión, al superar la idea de que lo individual y lo social son dúos opuestos en extremos diferentes de un espectro (Cole \& Wertsch, 1996), y verlos como interconectados, inseparables, e involucrados en una constante retroalimentación y determinación mutua (Overton, 2010). Esto es coherente con las conceptualizaciones del individuo en desarrollo actuando en relación con otros a través de prácticas culturales, un enfoque sistémico que pude ayudar a superar aspectos cuestionables de la psicología actual como la fragmentación, la cosificación, y suma de los datos de la gente (Raeff, 2017).

Las dimensiones como la individual-social operan a niveles múltiples de la metateoría, del dominio de la indagación a un nivel epistemológico más general (Overton, 2010). Por ello, la dimensión individual-social se refiere a las relaciones dinámicas entre un sujeto y un grupo, pero 
también a los conceptos de individualidad y sociabilidad en las teorías culturales que están en tensión entre sí. Estas dimensiones no se presentan como categorías opuestas propias de los paradigmas cartesianos, sino como puntos de vista (Latour, 1993), dimensiones continuas (Raeff, 2011), o niveles de análisis (Overton, 2010). Estas dimensiones culturales son articuladas por procesos culturales.

Los sistemas son una red relacional de procesos en los que el todo determina la naturaleza de los procesos y los procesos determinan la naturaleza del todo (Overton 2015). Por ejemplo, en la dimensión individual-social, el sujeto y el grupo no son vistos como contradicciones exclusivas, sino como polaridades únicas pero equivalentes de una relación integrada y comprometida en un ciclo de retroalimentación y determinación mutua (Overton, 2010). Los procesos culturales son creencias, valores, y lineamientos para la acción que son compartidos y desafiados, reflejados en lo que la gente hace, y en las herramientas culturales o formas de hacer esta acción posible (Wertsch, 1998). Los procesos culturales conectan a la gente, los lugares, y las prácticas. Abarcan las formas simbólicas de comunicación como acción (e.g., lenguaje), las dinámicas económicas y políticas, la organización del poder y la autoridad (Raeff, 2017), y los espacios institucionales para las prácticas culturales (Raeff, 2011).

Los procesos culturales en la dimensión individual-social crean y son creados por los sistemas culturales a través del tiempo. Esto es ejemplificado en el desarrollo de la identidad étnica-racial. Los padres miembros de minorías raciales y étnicas es Estados Unidos socializan a sus hijos a través de la discusión de lo que significa pertenecer a un grupo, lo que puede protegerlos de los efectos del estrés racial y la discriminación (Anderson \& Stevenson, 2019). En cambio, en las familias blancas la socialización parental típicamente consiste en evitar discutir o incluso reconocer temas que tienen que ver con la raza (Pahlke, Bigler, \& Suizzo, 2012). Esta 
socialización facilita el desarrollo de la identidad étnica-racial, los valores y las actitudes relacionadas con la importancia y el significado de la propia etnicidad y raza (Umaña-Taylor et al., 2014). Los procesos culturales se desarrollan con el tiempo, no simplemente mediante el cambio, pero al seguir una secuencia de diferenciación e integración progresiva hacia las metas del desarrollo (Raeff, 2011).

Segundo, la teoría y la investigación de la cultura como lugares refleja la tensión entre el tiempo y el espacio. Las teorías ecológicas describen cómo el cambio contextual a través del tiempo moldea el desarrollo (Bronfenbrenner, 1979; 2005). La naturaleza física de la cultura es acentuada en la investigación contextualista acerca de las implicaciones para el desarrollo de los padres con pocos ingresos que tienen dificultades para proveer juguetes, libros, y otros materiales didácticos para sus niños en el hogar, así como guarderías y escuelas de calidad, y/o vivir en vecindarios seguros (Duncan et al., 2017). De acuerdo con esta perspectiva, el individuo y su entorno son inseparables y están íntimamente conectados (Wapner \& Demick, 1998).

Pero la cultura también está enraizada en el tiempo, con diferentes generaciones estando expuestas a influencias únicas que dan forma a los valores que suscriben y a las prácticas que ejercen (Gentile, Campbell, \& Twenge, 2014). Hay evidencia científica que sugiere que hay importantes diferencias generacionales como resultado de experimentar adversidad económica en diferentes etapas del desarrollo. Comparados con sus hermanos mayores que se graduaron de la escuela antes de la recesión del 2007, los jóvenes que se graduaron durante esta crisis eran menos propensos a iniciar estudios universitarios (Pérez-Brena, Wheeler, Rodríguez De Jesús, Updegraff, \& Umaña-Taylor, 2017). Hay cambio, pero también continuidad, dentro del tiempo y el espacio. 
La dimensión temporal-espacial de la cultura reconcilia esta tensión, al abandonar la idea de que el tiempo y el espacio son dúos opuestos en polos extremos de un espectro, y verlos como entretejidos, inseparables, e involucrados en una constante retroalimentación y determinación mutua. Los procesos temporales-espaciales crean y son creados por los sistemas culturales. Por ejemplo, la disonancia ecológica entre generaciones ocurre debido a la exposición, acceso, y participación diferencial en contextos de aprendizaje en tiempos y espacios diferentes. Esto puede conducir a una tensión generacional entre cualquier grupo de personas, ya sean mayorías o minorías, migrantes o nativos.

La teoría de la angustia de la brecha de aculturación expone la discordancia, entre generaciones de padres de origen inmigrante y sus hijos, en la manera en que adoptan la cultura dominante y la cultura del país de origen (Telzer, 2010). Esta teoría argumenta que los hijos aprenden la cultura dominante de forma más rápida y extensa que sus padres (Szapocznik \& Kurtines, 1993), ya que los jóvenes suelen ser mejores que sus padres en aprender un idioma nuevo porque con frecuencia participan en prácticas de aprendizaje en espacios educativos en el país nuevo, durante tiempos de su desarrollo en el que son más propensos a aprender e identificarse con una cultura nueva (Gonzales et al., 2018).

La dimensión temporal-espacial de la cultura está alineada con una perspectiva sistémica que encuadra el tiempo y el espacio cono componentes interrelacionados que se moldean uno al otro y que no tienen sentido solos (Overton, 2010). El tiempo y el espacio son inseparables y centrales para una perspectiva de la cultura desde el desarrollo: "en lugar de invocar las formas abstractas y generalizables que la acción asume, el contextualismo se fundamenta en el ahora, en el momento, en las actividades en tiempo real de los organismos en escenarios y contextos específicos" (Witherington, 2007, p. 131). 
Tercero, la teoría e investigación en la cultura como prácticas refleja la tensión entre las conductas y los símbolos. Muchas conceptualizaciones de la cultura subrayan su naturaleza comportamental. La participación en prácticas comunitarias cotidianas es un aspecto central de la cultura (Raeff, 2006, 2017). Por ejemplo, la forma en que las madres leen libros en casa con sus niños pequeños y participan en la narración de historias puede promover el desarrollo del vocabulario y las habilidades narrativas (Luo \& Tamis-LeMonda, 2017).

Pero las prácticas también son simbólicas, incluyendo las ideologías que son transmitidas de una generación a la otra y que tienen un impacto considerable en el sentido de agencia y las interacciones sociales (Kendal, 2012). Los símbolos incluyen una variedad de conocimiento codificado en libros, fotos, videos, registros electrónicos, y las estructuras simbólicas encarnadas en leyes, normas, convenciones, e instituciones (Odling-Smee \& Laland, 2011). Por ejemplo, las meta-narrativas morales que funcionan como marco de referencia simbólico que proveen significado y motiva la conducta y la acción (Causadias, Updegraff, \& Overton, 2018).

La dimensión conductual-simbólica de la cultura reconcilia esta tensión, al superar la idea que los comportamientos y los símbolos son dúos opuestos en polos extremos de un espectro, a verlos como entretejidos, inseparables, e involucrados en una constante retroalimentación y determinación mutua. Los procesos conductuales-simbólicos crean y son creados por sistemas culturales. Esto es ilustrado en el caso de las tradiciones compartidas por los grupos como los ritos de iniciación, los rituales que marcan y celebran transiciones del desarrollo importantes de individuos dentro de sus comunidades (Rogoff, 2003). La participación en estos rituales tiene implicaciones para el desarrollo de la salud y la adaptación. Por ejemplo, las diadas de madres e hijas de origen mejicano que muestran mayor toma de decisiones compartida y comunicación 
franca después de celebrar la fiesta de "La Quinceañera" (Romo, Mireles-Rios, \& Lopez-Tello, 2014).

La acción es fundamental para entender los procesos culturales como este rito de iniciación, no como algo que un individuo o grupo poseen, pero algo que personifican (Raeff, 2017). "La Quinceañera" es una práctica de una gente en unos lugares que es creada por y crean una forma de cultura. Encarna la dimensión conductual-simbólica al personificar el significado psicológico a través de acciones intencionales representadas en el cuerpo (Müller \& Newman, 2008). Estas ideas están alineadas con conceptualizaciones de la cultura que enmarcan el comportamiento humano como simbólico (Geertz, 1973).

En conclusión, la consideración de las dimensiones y los procesos culturales en la definición de la cultura pude ayudar a hacer este concepto más crujiente y menos difuso: la cultura es un sistema, una organización dinámica que articula a la gente, lugares, y prácticas en un todo coherente a través de las dimensiones y los procesos individuales-sociales, temporalesespaciales, y conductuales-simbólicos.

\section{¿Por qué Hay Cultura? Entendiendo el modelo-p}

La cultura es un sistema de gente, lugares, y prácticas que se sostienen a través de dimensiones y procesos. Pero ¿con qué propósito? Yo argumento que el poder es una característica central de la cultura como sistema, y es útil en comprender por qué existe y persiste, y por qué es personificada, justificada, o combatida (Figura 1). Hay una larga tradición de debatir el propósito de la cultura. Como aspecto central de la evolución humana, la cultura es más que una simple operación funcional al servicio de la adaptación (ver Gould \& Lewontin, 1979). No hay certeza acerca del motivo por el cual algunos sistemas culturales persisten a través 
del tiempo, incluso cuando son onerosos o incluso dañinos para los individuos y los grupos, y por qué otras prácticas son abandonadas, a pesar de ser beneficiosas.

Una explicación sobre la continuidad en prácticas que no tienen una evidente función adaptativa es que tienen un propósito simbólico y proveen cohesión social al grupo (Northover \& Cohen, 2018). La cultura puede servir para organizar comunidades para resolver problemas comunes, como el desarrollo de tradiciones agriculturales para la supervivencia. Cuando algunos grupos ganan el control de recursos y tierra, con el tiempo, el poder puede llegar a ser el propósito de la cultura. En las democracias occidentales, especialmente aquellas derivadas del colonialismo, los propósitos de la cultura pueden ir más allá del mantenimiento de la cohesión grupal y pueden servir para ejercer el poder sobre otros grupos (Belich, 2009).

Pero ¿qué es el poder? Como la cultura, el poder también puede ser considerado un concepto difuso, invocado con frecuencia por científicos sociales, pero raramente definido con limites precisos (Roscigno, 2011). Hay una rica tradición de investigación sobre el poder en las ciencias sociales, incluyendo la psicología (Kraus \& Torrez, 2020) y la sociología (Roscigno, 2011). En sociología, la mejor forma de entender el poder es mediante las relaciones desiguales y asimétricas en sistemas estratificados imbuidos por la cultura y la historia (Bonilla-Silva, 2010). El poder provee un propósito, significado, y estructura a los sistemas culturales (Roscigno, 2011). Sorprendentemente, el poder también ha sido definido en términos de gente, lugares, y prácticas.

Una tradición clave en la definición del poder se enfoca en la gente: lograr que otros hagan lo que quieres que hagan, es decir, es un ejercicio de poder-sobre la gente (Allen, 2016; Foucault, 1983). El poder es social, ya que emerge de las relaciones desiguales, interactivas, y bilaterales entre la gente (Roscigno, 2011). La dimensión individual-social puede ilustrar el rol del poder en 
la cultura. Los sujetos con bastante poder pueden emplear herramientas discrecionales para afianzar su posición dominante en su grupo y para legitimar el tratamiento injusto de aquellos fuera del grupo invocando políticas y procedimientos (Roscigno, 2011), por ejemplo, haciendo cumplir "la ley y el orden" (Flamm, 2007). En cambio, individuos que sufren abusos de poder pueden unirse a otros y movilizar a sus grupos para luchar contra la desigualdad a través de la acción colectiva y la afirmación de la identidad, lo que constituye otra forma de poder (Neville, Viard, \& Turner, 2015).

Una segunda perspectiva para conceptualizar el poder se enfoca en los lugares: controlar el acceso a ambientes físicos, organizaciones, e instituciones. Es decir, el poder-en lugares. Esto conlleva el uso de la fuerza para mantener a la gente dentro o fuera de ciertos espacios en tiempos particulares, incluyendo vecindarios, escuelas, prisiones, empresas, y cualesquiera otras instituciones (García Coll et al., 1996). La dimensión temporal-espacial puede ilustrar el rol del poder en la cultura. A pesar del cambio aparente en algunas prácticas, las organizaciones pueden continuar siendo lugares impermeables en el ejercicio del poder que garantiza que las jerarquías se mantengan iguales (Roscigno, 2011). Las instituciones pueden invocar normas y regulaciones para reforzar la desigualdad, y al mismo tiempo, legitimar el orden establecido (Gramsci, 1971; Weber, 1978). Pero las organizaciones también pueden crear y ser creadas como lugares de resistencia, comprometidas en su misión de promover la igualdad y la justicia a través del tiempo y el espacio, como la Asociación Nacional para el Avance de la Gente de Color (en inglés, National Association for the Advancement of Colored People - NAACP; Sullivan, 2009).

Una tercera perspectiva para conceptualizar el poder se enfoca en las prácticas: la habilidad o capacidad para actuar y comportarse como es deseado, es decir, el poder-para practicar (Allen, 2016). Este enfoque está alineado con la definición clásica del poder formulada por Weber 
(1978): "la probabilidad de que un actor en una relación social esté en una posición de llevar a cabo su voluntad a pesar de la resistencia...” (p. 53). El concepto de poder está ligado a la habilidad para actuar: el latín potere y el francés pouvoir (Allen, 2016). La dimensión conductual-simbólica puede ilustrar el rol del poder en la cultura. El poder es tanto absoluto como relativo, real y percibido (Roscigno, 2011). Por esta razón, enfocarse en la desigualdad social como evidencia del desequilibrio de poder es importante pero insuficiente. Esto ignora situaciones en que el mero potencial o signo del poder es suficiente para recrear jerarquía (Roscigno, 2011). Pero los símbolos también pueden ser usados para protestar y resistir los abusos de poder, galvanizando la atención, y facilitando el cambio cultural, como el caso del saludo de Poder Negro en las olimpiadas de México de 1968 (Carlos \& Zirin, 2011), o cuando Colin Kaepernick y otros deportistas se arrodillaron durante el himno nacional de Estados Unidos en 2016 para protestar contra la brutalidad policial y el racismo.

La consideración del rol del poder en la definición de la cultura puede ayudar a hacerla un concepto más crujiente y menos difuso: la cultura es un sistema, una organización dinámica que articula gente, lugares, y prácticas en un todo coherente a través de dimensiones y procesos individuales-sociales, temporales-espaciales, y conductuales-simbólicos, con el propósito de afianzar, justificar, o resistir el poder-sobre la gente, el poder-en lugares, y el poder-para practicar.

\section{¿Por qué el Poder es Importante? Situando el modelo-p}

Reconocer el rol del poder en los sistemas culturales es un desafío para las ciencias aplicadas del desarrollo humano. Hay una aceptación creciente de la idea que los asuntos relacionados con el poder juegan un papel principal en el desarrollo de individuos que forman parte tanto de mayorías como de minorías, y de la importancia de la justicia y la equidad frente a la 
discriminación y exclusión social (Brown, Mistry, \& Yip, 2019; Killen, Rutland, \& Yip, 2016).

En los Estados Unidos, estos esfuerzos forman parte de una tradición de conceptualizaciones que intentan hacer que las ciencias aplicadas del desarrollo estén mejor conectadas con la realidad. Por ejemplo, el modelo integrativo para el estudio de las competencias del desarrollo en niños de minorías, en el cual García Coll y colegas (1996) situó el poder en el núcleo central de nuestra comprensión del desarrollo infantil (Causadias \& Umaña-Taylor, 2018). Esto es necesario debido a que muchos marcos conceptuales habían desatendido el rol de la estratificación social, el racismo, la opresión, y la segregación (García Coll et al., 1996).

Negar el papel central del poder en la cultura es una postura alineada con la evasión de poder, un aspecto central de las ideologías de daltonismo racial (o ceguera de color racial) que rechazan la existencia de problemas estructurales como el racismo al enfatizar la igualdad de oportunidades (Neville, Awad, Brooks, Flores, \& Bluemel, 2013). De acuerdo con este enfoque, los norteamericanos viven en una sociedad post-racial, las desigualdades se pueden explicar a partir de la falta de esfuerzo y mérito individual, y no deberíamos enfocarnos en la raza sino en el trabajo duro y la superación de adversidades (Bonilla-Silva, 2010). Utilizando esta idea en las ciencias aplicadas del desarrollo humano, un modelo que ubica el poder como uno de los propósitos de la cultura puede ser acusado de falta de rigor científico y objetividad, y que es motivado por una agenda política radical. Pero esta posición es, en sí misma, un ejercicio de poder de parte de los científicos del desarrollo, ya que ellos deciden que es considerado como ciencia y que no lo es. Es decir, "un aspecto del poder es la habilidad de determinar que cuenta como conocimiento y hacer que este conocimiento sea visto como 'natural' en lugar de verlo como una construcción humana" (Gjerde, 2004, p. 145). 
La idea de que el poder es un aspecto central de la cultura de Estados Unidos tiene el apoyo de la evidencia científica. En este país, la discriminación laboral de minorías raciales y étnicas con el mismo nivel de educación que personas blancas que aplican a los mismos trabajos ha mejorado poco en las últimas décadas (Quillian, Pager, Hexel, \& Midtbøen, 2017). En comparación con sus pares blancos, los hombres Afroamericanos, Indígenas, y Latinos tienen mayor riesgo a lo largo de su vida de ser matados por la policía (Edwards, Lee, \& Esposito, 2019). Para los hombres entre 25 y 35 años que son minorías, los homicidios por parte de la policía están entre las principales causas de muerte (Edwards et al., 2019). Estos hallazgos ilustran la existencia y persistencia del racismo, un sistema que muestra la centralidad del poder en la cultura.

\section{¿Qué es el Racismo? Ilustrando el modelo-p}

Hasta aquí, el modelo- $p$ ha sido presentado como una conceptualización abstracta. Para ilustrar su utilidad para las ciencias aplicadas del desarrollo humano, empleo este modelo para entender el racismo. Enmarcar el racismo como un sistema cultural es coherente con un rico legado de teoría e investigación (DiAngelo, 2018). El racismo es "la ideología racial de un sistema social racializado" (Bonilla-Silva, 2010, p. 218). En los Estados Unidos, la supremacía blanca es la forma dominante del racismo en el que la gente de descendencia europea reclama el derecho de subordinar aquellos que son de descendencia africana, asiática, latina, y/o indígena (Feagin, 2013). El racismo como sistema cultural requiere especial atención por parte de los científicos del desarrollo debido a su impacto en todos los niños, jóvenes, y sus familias (Seaton, Gee, Neblett, \& Spanierman, 2018).

El modelo-p puede ser usado para mostrar cómo las prácticas juegan un papel central en el mantenimiento de un sistema cultural como el racismo. El racismo en los Estados Unidos se 
mantiene a partir de la práctica de poner gente en lugares con el propósito de ejercer el poder. Esta práctica muestra una sorprendente continuidad en el tiempo y el espacio a través del confinamiento forzado de Indígenas norteamericanos en reservas indias, el cautiverio forzado de Afroamericanos en plantaciones de esclavos, la privación de libertad de norteamericanos de origen japonés en campos de concentración, la encarcelación masiva de hombres Latinos y Afroamericanos en cárceles y prisiones, el trato cruel de los refugiados centroamericanos en centros de detención, y la prohibición de la gente de países del medio oriente y África del norte de ingresar a Estados Unidos. Esta tradición es la puesta en práctica de ideas racistas (Kendi, 2017).

El modelo-p puede ser usado para entender el racismo como un sistema cultural encarnado en prácticas y conductas-símbolos. Cox (2003) examinó el rol de las Hijas Unidas de la Confederación, una asociación fundada en 1894, en el mantenimiento de la cultura confederada en los Estados Unidos. Cox (2003) documentó como lograron efectivamente moldear las percepciones y el comportamiento de generaciones de norteamericanos del sur a promover la meta-narrativa de la "Causa Perdida", la falsa historia de cómo la justa y heroica confederación defendió los derechos estatales del orgulloso estilo de vida del sur contra los agresores norteños, minimizando el papel central de la esclavitud en la guerra civil o guerra de secesión norteamericana (1861-1865). Las Hijas lograron dar una continuidad cultural a este mito mediante prácticas, incluyendo la enseñanza de esta narrativa en libros de texto revisionistas en las escuelas (Cox, 2003).

El modelo- $p$ puede ser usado para entender el racismo como un sistema cultural enraizado en lugares que mantienen tanto continuidad como cambio en tiempos-espacios. Las Hijas materializaron el mito de la meta-narrativa de la Causa Perdida mediante la transformación del 
paisaje norteamericano con monumentos entronizando como héroes a los criminales de guerra confederados (Cox, 2003). Si la confederación fue derrotada en la guerra civil o de secesión, las Hijas lograron una victoria cultural y ecológica en la preservación y redención de la supremacía blanca en Estados Unidos (Cox, 2003).

El modelo-p puede ser empleado para enfocar el racismo como un sistema cultural que afecta a la gente y daña a individuos-grupos. El legado cultural y ecológico de las Hijas no es una simple curiosidad histórica. Su efecto resuena hasta el día de hoy, afectando la vida de generaciones presentes mucho tiempo después del fallecimiento de las Hijas. Heather Heyer fue asesinada en un ataque terrorista durante la manifestación de "Unidad de la Derecha" el 12 de agosto de 2017 en Charlottesville, Virginia. El fin explícito de esta demostración de supremacistas blancos fue protestar contra el retiro de la estatua de Robert E. Lee, el comandante del ejército de los estados confederados. La afirmación de este legado ecológico a través de la violencia es un proceso que mantiene al racismo como el sistema cultural dominante en Estados Unidos.

El modelo-p puede ser empleado para enfocar el racismo como un sistema mantenido por procesos culturales. El proceso cultural de la construcción de mitos a través de la propagación de la meta-narrativa de la Causa Perdida también es ejemplo de transformación ecológica de ciudades y parques en altares de héroes de la confederación. Estas narrativas culturales cambian en el tiempo y el espacio, pero continúan influyendo en las conductas y los símbolos, lastimando individuos y grupos. En parte, la meta-narrativa de la Comunidad Perdida impulsa la ola creciente de movimientos de nacionalismo blanco, argumentando que había una vez comunidades tradicionales que vivían en armonía, pero la globalización, diversidad, y la inmigración destruyeron su cultura (Causadias et al., 2018a). Esta narrativa exacerba el 
sentimiento el sentimiento de amenaza de estatus entre la gente blanca en Estados Unidos que explica, en parte, la subida al poder de Donald J. Trump a la presidencia de Estados Unidos en 2016 (Mutz, 2018).

Finalmente, el modelo- $p$ se puede utilizar para enmarcar el racismo como un sistema cultural de poder y privilegio. Estas prácticas en lugares son implementadas para ejercer el poder sobre alguna gente (minorías raciales y étnicas) para mantener el privilegio de otras (gente blanca). En referencia al racismo, el poder-sobre la gente se traduce en dominación, la forma más extrema de poder (Blau, 1977). La dominación es el poder asimétrico ejercido de forma desenfrenada e injusta para controlar a otros y sus acciones (McCammon, 2018). Imperium (dominación por parte del estado) y dominium (dominación por parte de entidades privadas) son formas diferentes de dominación desde el tiempo de los romanos y su sistema republicano tradicional (Pettit, 1997, 2001). Esta distinción es útil para entender las formas contemporáneas de dominación racial, como el racismo patrocinado por el estado en el caso de la administración de Trump (Causadias, 2019), así como el racismo patrocinado por las corporaciones que lucran de la encarcelación masiva en Estados Unidos (Alexander, 2012).

En conclusión, el caso del racismo ilustra el modelo- $p$, demostrando como la gente, lugares, y prácticas; expresadas en dimensiones; mantenidas a través de procesos; crean y son creadas por un sistema. Un sistema que se convierte en algo más que una gente, un lugar, y una práctica. Una vez establecido, el racismo se convierte en una organización estable que es más que sus partes y no puede ser reducida fácilmente a una de ellas. Así, los sistemas culturales pueden ser considerados como unidades de análisis irreducibles.

\section{¿Cuál es la Utilidad de Esto? Implementando el modelo-p}


El modelo- $p$ puede tener aplicaciones útiles para la teoría, investigación, y aplicación en las ciencias del desarrollo. En teoría, definir la cultura como sistema destaca la naturaleza dinámica del intercambio entre gente, lugares, y prácticas. Las teorías de sistemas realzan el valor de entender las conexiones dinámicas entre diferentes componentes de un sistema (von Bertalanffy, 1976). Esto implica ir más allá de conceptualizaciones que separan los efectos causales de un componente particular (gente, lugares, o prácticas), para adoptar una nueva comprensión de cómo los sistemas operan como un todo dinámico (Diez Roux, 2011).

Esta comprensión tiene repercusiones fundamentales para las teorías del desarrollo, ya que transforma nuestro enfoque del desarrollo y la salud de los individuos y grupos, para ir de los factores aislados a las manifestaciones de un sistema (Diez Roux, 2011). Esto puede ser un desafío para el estudio de la cultura y el desarrollo individual, donde hay varios sistemas en juego. Por ejemplo, el racismo y el sexismo son sistemas culturales que se entrelazan para crear sinergias que los potencian a ambos, afectando el desarrollo y la adaptación de las mujeres negras de manera singular, ya que ellas experimentan desafíos que los hombres negros y las mujeres blancas no enfrentan (Allen, 2016; Crenshaw, 1991).

En investigación, el modelo- $p$ puede ser aplicado para el estudio de los sistemas culturales examinando las dinámicas poblacionales, ambientales, y participativas. Esto implica interrogar al sistema al examinar sus componentes. En el caso del racismo, esto implica considerar las dinámicas poblacionales (gente) a través de la evaluación individual, así como colectiva, de las experiencias de discriminación. Por ejemplo, esto se puede lograr midiendo la discriminación racial mediante enfoques subjetivos (percibida) y objetivos (estudios de auditoria; Seaton, et al., 2018). También conlleva examinar las dinámicas ecológicas (lugares) mediante la evaluación de contextos espaciales y temporales. Por ejemplo, evaluar dinámicas ecológicas puede incluir el 
uso de métodos geográficos (segregación residencial) e históricos (políticas gubernamentales y privadas de exclusión racial residencial). Implica investigar dinámicas participativas (prácticas) al evaluar como la gente se involucra conductual y simbólicamente en sus comunidades usando enfoques cuantitativos (cuestionarios) y cualitativos (etnografías).

El modelo-p puede ser aplicado a la investigación mediante la creación de matrices multirasgo y multi-método que evalúen distintas dimensiones y procesos culturales con diferentes medidas e instrumentos de evaluación (Campbell \& Fiske, 1959). Además, un enfoque sistémico en la investigación de la cultura requiere superar la doctrina del individualismo metodológico en las ciencias sociales, en el que los asuntos sociales son reducidos a datos sobre individuos (DiezRoux, 1998). Es fundamental buscar nuevas formas de evaluar sistemas culturales, no sólo individuos. Por ejemplo, Kleinman (1995) animó a los etnógrafos a examinar "niveles de análisis colectivo (tanto local como social) e individual (tanto público como íntimo)” (p. 98).

En la práctica, el modelo- $p$ puede ser aplicado en las ciencias del desarrollo humano en la educación, políticas públicas, e intervenciones. Por ejemplo, puede ser aplicado mediante el reconocimiento del rol del poder como uno de los propósitos de los sistemas culturales, especialmente al enfocar a los educadores, a los creadores de políticas publicas, y a los clínicos como actores poderosos. En la educación, esto implica reconocer como los profesores universitarios titulares se benefician de su posición dominante en los sistemas educativos, teniendo bastante poder-sobre los estudiantes. En las políticas, esto implica apreciar el rol privilegiado de los creadores de políticas publicas en decidir quién recibe acceso y cómo, teniendo bastante poder-en instituciones. En las intervenciones, esto significa reconocer cómo aquellos diseñando e implementando estos programas deciden qué conductas son enmarcadas como normales o anormales, necesitando modificación y tratamiento, escogiendo algunos grupos 
como el estándar de la salud y el criterio base para las comparaciones (Sroufe, 1970). Ellos tienen poder-para intervenir.

Hacer un sistema cultural más equitativo requiere compartir el poder y la toma de decisiones con los estudiantes en la educación, con los ciudadanos en la creación de políticas públicas, y con los miembros de la comunidad en las intervenciones. Los educadores, creadores de políticas públicas, y clínicos también pueden usar su poder para desafiar y resistir los sistemas culturales. Por ejemplo, al alejarse de los modelos de déficit de las minorías raciales y étnicas para promover el desarrollo positivo de los jóvenes (Gaylord-Harden, Barbarin, Tolan, \& Murry, 2018), y aprovechar el potencial sanador de la socialización racial (Anderson \& Stevenson, 2019). Además, un enfoque sistémico del poder como uno de los propósitos de la cultura exige aceptar que éste no se puede reducir a un solo asunto que abordar mediante la educación, las políticas públicas, o las intervenciones. Es decir, “el poder no se puede reducir teóricamente a un atributo singular, una posición, o a una simple ecuación de costos, beneficios, y balances o desequilibrios tangibles" (Roscigno, 2011, p. 353). Las prácticas justas deben apuntar al cambio del sistema como un todo.

\section{Limitaciones y Direcciones Futuras}

La cultura es un concepto difuso, lo que hace difícil definirla. En este artículo, introduje un modelo- $p$ para hacer la cultura un concepto más crujiente y menos difuso: un sistema de gente, lugares, y prácticas, con un propósito como afianzar, justificar, o resistir el poder. Sin embargo, hay varias limitaciones de este modelo que requieren mayor atención y refinamiento, y varios asuntos importantes que no he atendido.

Primero, es improbable que este modelo pueda superar la difícil tarea de definir la cultura y llegar a un acuerdo en torno a su definición. Al final, el problema "es que nadie está seguro de 
qué es la cultura...es [un término] fugitivo, inestable, enciclopédico, y cargado de normatividad... [alguna gente] piensa que es [un término] vacío, e incluso peligroso, y que lo deberían prohibir de los debates serios de la gente seria” (Geertz, 2000, p. 11).

Segundo, hay muchos más componentes relevantes de la cultura que yo no discutí detalladamente que merecen más atención en el futuro, incluyendo los productos cultuales y las herramientas (Gibson, Gibson, \& Ingold, 1994). A pesar de este alcance limitado, enfocarse en la gente, lugares, y prácticas puede ayudar a los investigadores a clarificar lo que quieren decir cuando usan el término cultura en la teoría, investigación, e intervenciones. Adicionalmente, he enmarcado este modelo en teoría e investigación de los Estados Unidos de América, a pesar de las desventajas que esto conlleva (Arnett, 2008). Más atención debe prestarse a entender hasta qué punto este modelo es aplicable a otros países.

Tercero, hay muchas más dimensiones y procesos importantes que yo no discutí detalladamente que merecen mayor atención en el futuro, incluyendo las dimensiones internasexternas, cercanas-distantes, relativas-universales, y objetivas-subjetivas de la cultura. A pesar de su alcance limitado, enfocarse en las dimensiones y procesos individuales-sociales, temporalesespaciales, y conductuales-simbólicos pueden ser útil en cómo pensamos acerca de la cultura, no sólo en cómo la medimos. Por ejemplo, la dimensión individual-social va más allá de medir ambos aspectos. Además de enfocarse en uno (individual $o$ social) o ambos (individual $y$ social), una perspectiva sistémica conlleva pensar como se construyen mutuamente: como lo individual es social y como lo social es individual. Lo mismo es cierto para las dimensiones temporalesespaciales y conductuales-simbólicas.

Cuarto, enmarcar la cultura como un sistema con un propósito como sostener el poder-sobre la gente, el poder-en lugares, y el poder-para practicar puede brindar una visión incompleta. Esta 
perspectiva puede soslayar casos en el que el poder no es el propósito central de la cultura. Por ejemplo, la cultura puede proveer significado, como ilustra el desarrollo de un propósito de vida integrado, resiliente, y articulado entre adolescentes que experimentan marginalización (Sumner, Burrow, \& Hill, 2018). Adicionalmente, el poder no es necesariamente opresivo, pero puede tener un rol normativo y beneficioso, e incluso ser liberador. Hay características centrales de la cultura, como el lenguaje, que pueden ser emancipadoras en lugar de injustas (Vygotsky, 1997).

\section{Conclusión}

Creo que entender la cultura como sistema puede ser útil para mejorar el trabajo aplicado en las ciencias del desarrollo humano. Los estudios, herramientas de evaluación, políticas públicas, e intervenciones enfocadas en gente, lugares, y prácticas específicas son fundamentales. Ellas pueden crear conexiones preliminares entre componentes de redes más amplias, avanzando gradualmente nuestra comprensión del sistema completo (Kendler, 2005). Al mismo tiempo, debemos tener en cuenta que estos esfuerzos solo encaran partes de un sistema mucho más grande y no brindan una perspectiva completa de lo que es la cultura. Para lograr el cambio cultural necesario para superar el racismo se requiere atender el sistema completo, así como sus partes. Si no estamos a la altura de este desafío, corremos el riesgo de continuar siendo una ciencia del "entusiasmo y limonada rosada" (Cronbach, 1957, p. 671). Si esto pasa, los conceptos difusos van a ser el menor de nuestros problemas. 


\section{Lista de referencias}

Alcalá, L., Rogoff, B., Mejía-Arauz, R., Coppens, A. D., \& Dexter, A. L. (2014). Children's initiative in contributions to family work in indigenous-heritage and cosmopolitan communities in Mexico. Human Development, 57(2-3), 96-115.

Alexander, M. (2012). The New Jim Crow: Mass Incarceration in the Age of Colorblindness. New York: The New Press.

Allen, A. (2016). Feminist perspectives on power. In E. N. Zalta (Ed.), The Stanford Encyclopedia of Philosophy (Fall 2016 Edition). Retrieved from https://plato.stanford.edu/archives/fall2016/entries/feminist-power/

Anderson, R. E., \& Stevenson, H. C. (2019). RECASTing racial stress and trauma: Theorizing the healing potential of racial socialization in families. American Psychologist, 74(1), 63.

Arnett, J. J. (2008). The neglected 95\%: Why American psychology needs to become less American. American Psychologist, 63(7), 602-614. doi:10.1037/0003-066X.63.7.602

Belich, J. (2009). Replenishing the earth: The settler revolution and the rise of the Angloworld. New York: Oxford University Press.

Betancourt, H., \& López, S. R. (1993). The study of culture, ethnicity, and race in American psychology. American Psychologist, 48(6), 629-637.

Betzig, L. (2015). Culture. In J. Brockman (Ed.), This idea must die. Scientific theories that are blocking progress (pp. 429-431). New York: Harper Perennial.

Blau, P. M. (1977). Inequality and heterogeneity: A primitive theory of social structure (Vol. 7). New York: Free Press.

Bonilla-Silva, E. (2010). Racism without racists: Color-blind racism and the persistence of racial inequality in the United States. Rowman \& Littlefield Publishers. 
Boyer, P. (2015). Culture. In J. Brockman (Ed.), This idea must die. Scientific theories that are blocking progress (pp. 426-428). New York: Harper Perennial.

Bronfenbrenner, U. (1979). The ecology of human development. Cambridge: Harvard University Press.

Bronfenbrenner, U. (2005). Making human beings human: Bioecological perspectives on human development. Thousand Oaks, CA: Sage Publications.

Brown, C. S., Mistry, R. S., \& Yip, T. (2019). Moving from the margins to the mainstream: equity and justice as key considerations for developmental science. Child Development Perspectives, 13(4), 235-240.

Campbell, D. T., \& Fiske, D. W. (1959). Convergent and discriminant validation by the multitrait-multimethod matrix. Psychological Bulletin, 56(2), 81.

Carlos, J., \& Zirin, D. (2011). The John Carlos Story: The Sports Moment That Changed the World. New York: Haymarket Books.

Causadias, J. M. (2013). A roadmap for the integration of culture into developmental psychopathology. Development and Psychopathology, 25(4pt2), 1375-1398.

Causadias, J. M. (2019). Are you researching the effects of the Trump administration's statesponsored racism? Please call it by its name! Medium: National Center for Institutional Diversity. Retrieved from: https://medium.com/national-center-for-institutionaldiversity/are-you-researching-the-effects-of-the-trump-administrations-state-sponsored$\underline{\text { racism-5acab7d4ad3d }}$

Causadias, J. M., \& Umaña-Taylor, A. J. (2018). Reframing marginalization and youth development: Introduction to the special issue. American Psychologist, 73(6), 707-712. American Psychologist. doi:10.1037/amp0000336 
Causadias, J. M., Updegraff, K. A., \& Overton, W. F. (2018a). Moral meta-narratives, marginalization, and youth development. American Psychologist, 73(6), 827-839.

Causadias, J. M., Vitriol, J. A., \& Atkin, A. L. (2018b). Do we overemphasize the role of culture in the behavior of racial/ethnic minorities? Evidence of a cultural (mis)attribution bias in American Psychology. American Psychologist, 73(3), 243-255.

Cicchetti, D. (1984). The emergence of developmental psychopathology. Child Development, 55, 1-7. doi: $10.2307 / 1129830$

Cole, M. (1979). Foreword. In U. Bronfenbrenner, The ecology of human development: Experiments by nature and design. Cambridge, MA: Harvard University Press.

Cole, M., Gay, J., Glick, J., \& Sharp, D. (1971). The cultural context of learning and thinking: An exploration in experimental anthropology. New York: Basic Books.

Cole, M., \& Wertsch, J. V. (1996). Beyond the individual-social antinomy in discussions of Piaget and Vygotsky. Human development, 39(5), 250-256.

Cooper, C. R., \& Denner, J. (1998). Theories linking culture and psychology: Universal and community-specific processes. Annual Review of Psychology, 49(1), 559-584.

Cox, K. L. (2003). Dixie's Daughters: The United Daughters of the Confederacy and the Preservation of Confederate Culture. University Press of Florida.

Crenshaw, K. (1991). Mapping the margins: Intersectionality, identity politics, and violence against women of color. Stanford Law Review, 43(6), 1241-1299.

Cronbach, L. J. (1957). The two disciplines of scientific psychology. American Psychologist, 12, 671-684. doi:10.1037/h0043943

DiAngelo, R. (2018). White fragility: Why it's so hard for white people to talk about racism. Beacon Press. 
Diez Roux, A. V. (2011). Complex systems thinking and current impasses in health disparities research. American Journal of Public Health, 101(9), 1627-1634.

Diez-Roux, A. V. (1998). Bringing context back into epidemiology: variables and fallacies in multilevel analysis. American Journal of Public Health, 88(2), 216-222.

Duncan, G. J., Magnuson, K., \& Votruba-Drzal, E. (2017). Moving beyond correlations in assessing the consequences of poverty. Annual Review of Psychology, 68, 413-434.

Durham, W. H. (1991). Coevolution: Genes, culture, and human diversity. Stanford: Stanford University Press.

Edwards, F., Lee, H., \& Esposito, M. (2019). Risk of being killed by police use of force in the United States by age, race-ethnicity, and sex. Proceedings of the National Academy of Sciences, 116(34), 16793-16798.

Fausset, R., \& Feuer, A. (2017). Far-Right groups surge into national view in Charlottesville. The New York Times. Retrieved from: https://web.archive.org/web/20170816015808/https://www.nytimes.com/2017/08/13/us/f ar-right-groups-blaze-into-national-view-in-charlottesville.html

Feagin, J. (2013). Systemic racism: A theory of oppression. New York: Routledge.

Flamm, M. W. (2007). Law and order: Street crime, civil unrest, and the crisis of liberalism in the 1960s. Columbia University Press.

Foucault, M. (1983). Afterword: The subject and power. in H. Dreyfus and P. Rabinow (Eds.), Michel Foucault: Beyond Structuralism and Hermeneutics, 2nd edition. Chicago: University of Chicago Press.

Fuller, B., \& García Coll, C. (2010). Learning from Latinos: Contexts, families, and child development in motion. Developmental Psychology, 46, 559-565. 
García Coll, C., Lamberty, G., Jenkins, R., McAdoo, H. P., Crnic, K., Wasik, B., \& García, H. V. (1996). An integrative model for the study of developmental competencies in minority children. Child Development, 67, 1891-1914.

Gaylord-Harden, N. K., Barbarin, O., Tolan, P. H., \& Murry, V. M. (2018). Understanding development of African American boys and young men: Moving from risks to positive youth development. American Psychologist, 73(6), 753-767. doi:10.1037/amp0000300

Geertz, C. (1973). Interpretation of cultures: Selected essays by Clifford Geertz. New York: Basic Books.

Geertz, C. (2000). Available light: Anthropological reflections on philosophical topics. Princeton, NJ: Princeton University Press.

Gentile, B., Campbell, W. K., \& Twenge, J. M. (2014). Generational cultures. In A. B. Cohen (Ed.), Culture reexamined: Broadening our understanding of social and evolutionary influences (pp. 31-48). Washington, DC, US: American Psychological Association.

Gibson, K. R., Gibson, K. R., \& Ingold, T. (1994). Tools, language and cognition in human evolution. Cambridge University Press.

Gjerde, P. F. (2004). Culture, power, and experience: Toward a person-centered cultural psychology. Human Development, 47(3), 138-157.

Gonzales, N. A., Knight, G. P., Gunn, H. J., Tein, J. Y., Tanaka, R., \& White, R. M. (2018). Intergenerational gaps in Mexican American values trajectories: Associations with parent-adolescent conflict and adolescent psychopathology. Development and Psychopathology, 30(5), 1611-1627. doi:10.1017/S0954579418001256 
Gould, S. J., \& Lewontin, R. C. (1979). The spandrels of San Marco and the Panglossian paradigm: A critique of the adaptationist programme. Proceedings of the Royal Society of London. Series B. Biological Sciences, 205(1161), 581-598. doi:10.1098/rspb.1979.0086

Gramsci, A. (1971). Selections from the Prison Notebooks. In Q. Hoare and G.N. Smith (Eds.). International Publishers.

Haack, S. (1996). Deviant logic, fuzzy logic: Beyond the formalism. Chicago: University of Chicago Press.

Kendal, J. (2012). Cultural niche construction and human learning environments: Investigating sociocultural perspectives. Biological Theory 6(3), 241-250.

Kendi, I. X. (2017). Stamped from the beginning: The definitive history of racist ideas in America. New York: Random House.

Kendler, K. S. (2005). Toward a philosophical structure for psychiatry. American Journal of Psychiatry, 162(3), 433-440. doi:10.1176/appi.ajp. 162.3.433

Killen, M., Rutland, A., \& Yip, T. (2016). Equity and justice in developmental science: Discrimination, social exclusion, and intergroup attitudes. Child Development, 87, 13171336. https://doi.org/10. 1111/cdev.12593

Kitayama, S., \& Uskul, A. K. (2011). Culture, mind, and the brain: Current evidence and future directions. Annual Review of Psychology, 62, 419- 449.

Kleinman, A. (1995). Writing at the margin: Discourse between anthropology and medicine. Berkeley: University of California Press.

Kraus, M., \& Torrez, B. (2020). A psychology of power that is embedded in societal structures. Current Opinion in Psychology, 33, 86-90. doi:10.1016/j.copsyc.2019.07.018 
Kroeber, A. L., \& Kluckhohn, C. (1952). Culture: A critical review of concepts and definitions. Papers. Peabody Museum of Archaeology \& Ethnology, Harvard University.

Latour, B. (1993). We have never been modern. Cambridge, MA: Harvard University Press.

Lonner, W. J., \& Malpass, R. S. (1994). When psychology and culture meet: An introduction to cross-cultural psychology. In W. J. Lonner \& R. S. Malpass (Eds.), Psychology and culture (pp. 1-12). Boston, MA: Allyn \& Bacon.

Luo, R., \& Tamis-LeMonda, C. S. (2017). Reciprocity between maternal questions and child contributions during book-sharing. Early Childhood Research Quarterly, 38, 71-83.

Markus, H. R., \& Kitayama, S. (2010). Cultures and selves: A cycle of mutual constitution. Perspectives on Psychological Science, 5(4), 420-430.

McCammon, C. (2018). Domination. In E. N. Zalta (Ed.), The Stanford Encyclopedia of Philosophy (Winter 2018 Edition). Retrieved from: https://plato.stanford.edu/archives/win2018/entries/domination/

Müller, U., \& Newman, J. L. (2008). The body in action: Perspectives on embodiment and development. In W. F. Overton, U. Müller, \& J. L. Newman (Eds.), Developmental perspectives on embodiment and consciousness (pp. 313-342). New York, NY: Taylor \& Francis.

Mutz, D. C. (2018). Status threat, not economic hardship, explains the 2016 presidential vote. Proceedings of the National Academy of Sciences, 115(19), E4330-E4339.

Neville, H. A., Awad, G. H., Brooks, J. E., Flores, M. P., \& Bluemel, J. (2013). Color-blind racial ideology: Theory, training, and measurement implications in psychology. American Psychologist, 68(6), 455-466. 
Neville, H. A., Viard, B., \& Turner, L. (2015). Race and recognition: Pathways to an affirmative Black identity. Journal of Black Psychology, 41(3), 247-271.

Northover, S. B., \& Cohen, A. B. (2018). Understanding religion from cultural and biological perspectives. In J. M. Causadias, E. H. Telzer, \& N.A. Gonzales (Eds.), Handbook of Culture and Biology (pp. 55-77). New York: Wiley.

Odling-Smee, J., \& Laland, K. N. (2011). Ecological inheritance and cultural inheritance: What are they and how do they differ? Biological Theory, 6(3), 220-230.

Overton, W. F. (2010). Life-span development: Concepts and issues. In R. M. Lerner (Ed. in Chief) \& W. F. Overton (Vol. Ed.), Handbook of life-span development: Vol. 1. Cognition, biology, and methods across the lifespan (pp. 1-29). Hoboken, NJ: Wiley.

Overton, W. F. (2015). Processes, relations and relational-developmental-systems. In W. F. Overton \& P. C. M. Molenaar (Eds.). Handbook of child psychology and developmental science. Theory and Method. Volume (pp. 9-62) (7th ed.), Editor-in-Chief: Richard M. Lerner. Hoboken, NJ: Wiley.

Overton, W. F., \& Müller, U. (2012). Metatheories, theories, and concepts in the study of development. In R. M. Lerner, M. A. Easterbrooks, \& J. Mistry (Eds.), Developmental psychology. Volume 6 of the Handbook of psychology (pp. 19-58). Editor-in-Chief: I. B. Weiner. Hoboken, NJ: Wiley.

Pahlke, E., Bigler, R. S., \& Suizzo, M. A. (2012). Relations between colorblind socialization and children's racial bias: Evidence from European American mothers and their preschool children. Child Development, 83, 1164-1179. doi:10.1111/j.1467-8624.2012 .01770.x

Pérez-Brena, N. J., Wheeler, L. A., Rodríguez De Jesús, S. A., Updegraff, K. A., \& UmañaTaylor, A. Y. (2017). The educational and career adjustment of Mexican-origin youth in 
the context of the 2007/2008 economic recession. Journal of Vocational Behavior, 100, 149-163. doi:10.1016/j.jvb.2017.02.006

Pettit, P. (1997). Republicanism: A Theory of Freedom and Government. Oxford: Clarendon Press.

Pettit, P. (2001). A Theory of Freedom: From the Psychology to the Politics of Agency. Oxford: Oxford University Press.

Quillian, L., Pager, D., Hexel, O., \& Midtbøen, A. H. (2017). Meta-analysis of field experiments shows no change in racial discrimination in hiring over time. Proceedings of the National Academy of Sciences, 114(41), 10870-10875.

Quintana, S. M., Aboud, F. E., Chao, R. K., Contreras-Gray, J., Cross, W. E., Jr., Hurdley, C., et al. (2006). Race, ethnicity, and culture in child development: Contemporary research and future directions. Child Development, 77, 1129-1141.

Raeff, C. (2006). Individuals in relation to others: Independence and interdependence in a kindergarten classroom. Ethos, 34(4), 521-557.

Raeff, C. (2011). Distinguishing between development and change: Reviving organismicdevelopmental theory. Human Development, 54(1), 4-33.

Raeff, C. (2017). Going where the action is to conceptualize the person. New Ideas in Psychology, 44, 7-13.

Rogoff, B. (2003). The cultural nature of human development. New York: Oxford University Press.

Romo, L. F., Mireles-Rios, R., \& Lopez-Tello, G. (2014). Latina mothers' and daughters’ expectations for autonomy at age 15 (La Quinceañera). Journal of Adolescent Research, 29, 271-294. doi:10.1177/0743558413477199 
Roscigno, V.J. (2011). Power, revisited. Social Forces, 90, 349-374. doi: 10.1093/sf/sor034

Seaton, E. K., Gee, G. C., Neblett, E., \& Spanierman, L. (2018). New directions for racial discrimination research as inspired by the integrative model. American Psychologist, 73(6), 768-780. doi:10.1037/amp0000315

Shweder, R. A. (2000). The psychology of practice and the practice of the three psychologies. Asian Journal of Social Psychology, 3(3), 207-222. doi:10.1111/1467-839X.00065

Snowdon, C. T. (2018). Introduction to animal culture: Is culture uniquely human? In J. M. Causadias, E. H. Telzer, \& N.A. Gonzales (Eds.), Handbook of Culture and Biology (pp. 81-104). New York: Wiley.

Spencer-Oatey, H., \& Franklin, P. (2012). What is culture. A compilation of quotations. GlobalPAD Core Concepts, 1-22.

Sroufe, L. A. (1970). A methodological and philosophical critique of intervention-oriented research. Developmental Psychology, 2(1), 140-145. doi:10.1037/h0028598

Sue, S. (2009). Ethnic minority psychology: Struggles and triumphs. Cultural Diversity and Ethnic Minority Psychology, 15(4), 409-415.

Sullivan, P. (2009). Lift every voice: The NAACP and the making of the civil rights movement. New York: The New Press.

Sumner, R., Burrow, A. L., \& Hill, P. L. (2018). The development of purpose in life among adolescents who experience marginalization: Potential opportunities and obstacles. American Psychologist, 73(6), 740-752.

Super, C. M., \& Harkness, S. (1986). The developmental niche: A conceptualization at the interface of child and culture. International Journal of Behavioral Development, 9(4), $545-569$. 
Szapocznik, J., \& Kurtines, W. M. (1993). Family psychology and cultural diversity: Opportunities for theory, research, and application. American Psychologist, 48, 400-407.

Telzer, E. H. (2010). Expanding the acculturation gap-distress model: An integrative review of research. Human Development, 53(6), 313-340. doi:10.1159/000322476

Tooby, J. (2015). Learning and culture. In J. Brockman (Ed.), This idea must die. Scientific theories that are blocking progress (pp. 432-436). New York: Harper Perennial.

Triandis, H. C. (2007). Culture and psychology: A history of the study of their relationships. In S. Kitayama \& D. Cohen (Eds.), Handbook of cultural psychology (pp. 59-76). New York: Guilford Press.

Tylor, E. B. (1871). Primitive culture: Researches into the development of mythology, philosophy, religion, art, and custom, etc. London: Murray.

Umaña-Taylor, A. J., Quintana, S. M., Lee, R. M., Cross, W. E., Rivas-Drake, D., Schwartz, S. J., . . Ethnic and Racial Identity in the 21st Century Study Group. (2014). Ethnic and racial identity during adolescence and into young adulthood: An integrated conceptualization. Child Development, 85, 21-39.

von Bertalanffy, L. (1976). General system theory: Foundations, development, applications. New York, NY: George Braziller.

Vygotsky, L. S. (1997). The collected works of L.S. Vygotsky: Problems of the theory and history of psychology (Vol. 3). Springer Science \& Business Media.

Wapner, S., \& Demick, J. (1998). Developmental analysis: A holistic, developmental, systemsoriented perspective. In R.M. Lerner (Ed.), Theoretical models of human development. Handbook of child psychology. Vol. 4 (pp. 761-805). New York: Wiley. 
Weber, M. (1978). Economy and Society: An Outline of Interpretive Sociology, trans. Ephraim Fischoff et al. Berkeley, CA: University of California Press.

Wertsch, J.V. (1998). Mind as action. New York: Oxford University Press.

Witherington, D.C. (2007). The dynamic systems approach as metatheory for developmental psychology. Human Development, 50, 127-153. 